\title{
Sistema de selección de sistemas elastocompresivos en personas con cáncer de mama de un hospital con recursos limitados
}

\author{
Selection system for elastocompressive systems in people with breast cancer from \\ a hospital with limited resources
}

Williams Mella-Abarca', Valentina Barraza-Sánchez², Karol Ramírez-Parada ${ }^{3}$

\section{Resumen}

El cáncer de mama es un problema de salud pública en Chile. El linfedema es un trastorno linfovascular secundario a la extirpación de los ganglios linfáticos por cirugía en el cáncer de mama, que produce un aumento del volumen y la fibrosis en el miembro superior. Diferentes prendas de compresión son usadas para la prevención y el tratamiento del linfedema. Por eso, el programa de Garantías Explícitas en Salud garantiza la entrega de sistemas elastocompresivos a las personas con diagnóstico de cáncer de mama para prevenir y tratar el linfedema. Sin embargo, en hospitales públicos los sistemas elastocompresivos pueden ser recursos limitados, por eso, muchas veces se deben priorizar. Este artículo tiene por objetivo describir un sistema de selección de sistemas elastocompresivos en personas con diagnóstico de cáncer de mama en el modelo de atención kinesiológico temprano y prospectivo.

Palabras clave: neoplasia de mama; linfedema secundario a cáncer de mama; mangas elastocompresivas; tratamiento de linfedema; prevención de linfedema.

\begin{abstract}
Breast cancer is a public health problem in Chile. Lymphedema is a lymphovascular disorder secondary to the removal of lymph nodes by surgery in breast cancer, resulting in increased volume and fibrosis in the upper limb. Different compression garments are used for the prevention and treatment of lymphedema. Therefore, the "Garantías Explícitas en Salud" program guarantees the delivery of compression garments to people diagnosed with breast cancer to prevent and treat lymphedema. However, in public hospitals, the compression garments can be limited resources, so they often need to be prioritized. This article aims to describe the selection system for compression garments in people diagnosed with breast cancer in the early and prospective physical therapy care model.
\end{abstract}

Keywords: breast cancer; breast cancer lymphedema; compression garments; lymphedema treatment; lymphedema prevention.

Fecha de envío: 2020-05-21 - Fecha de aceptación: 2020-11-24

\section{Introducción}

En los hospitales públicos con recursos limitados es importante hacer un buen uso de los insumos de cualquier índole. En Chile, los sistemas elastocompresivos en personas con cáncer de mama están garantizados por las Garantías Explícitas en Salud en la fase de seguimiento (MINSAL, 2016; Ramírez et al., 2019). Muchas veces, para disminuir los costos en salud se seleccionan sistemas elastocompresivos inadecuados.
El objetivo de este artículo es describir una forma de clasificación clínica para la selección de sistemas elastocompresivos en personas con diagnóstico de cáncer de mama en el modelo de atención kinesiológico temprano y prospectivo (Ramírez et al., 2019).

\section{Cáncer de mama y linfedema secundario}

El cáncer de mama es un problema de salud pública en Chile y el mundo. En la población femenina es el cáncer diagnosticado con

(1) Servicio de kinesiología intensiva y soporte ventilatorio. Hospital de Urgencia y asistencia pública. Santiago, Chile.

(2) Departamento de Kinesiología. Casa Salvador (Chile). Santiago, Chile.

(3) Departamento de Kinesiología. Departamento de Ciencias de la Salud. Pontificia Universidad Católica de Chile. Santiago, Chile.

Autor de correspondencia: kramirezp@uc.cl 
Mella et al.

mayor frecuencia, con una incidencia de $24,2 \%$, y es la principal causa de muerte por cáncer con una mortalidad de 15\% (Bray et al., 2018). El tratamiento habitual de esta patología dependerá de su biología y estadificación. Los principales tratamientos incluyen cirugía, quimioterapia, radioterapia y terapia biológica (Ramírez et al., 2017). La cirugía puede producir diversas alteraciones secundarias, donde destaca el linfedema, síndrome de red axilar, limitación de movilidad y disminución de la fuerza muscular (Hidding et al., 2014)

El linfedema se define como un acumulo de agua, sales, electrolitos, proteínas de alto peso molecular y otros elementos en el espacio intersticial, que llevan a un aumento de volumen de la región corporal afectada. Esta alteración es consecuencia de una alteración dinámica y/o mecánica de la circulación linfática, que lleva a un aumento de volumen progresivo y evolutivo de la extremidad o región corporal. Si bien, el linfedema no tiene cura, puede ser prevenido y controlado (Rockson et al., 2019).

Existen diversos métodos para el diagnóstico del linfedema. Se pueden utilizar pruebas de laboratorio o imagenológicas como la linfocintigrafía isotópica con indocianina verde o sistemas similares (Pereira \& Koshima, 2018). Otra opción es utilizar la historia clínica, examen físico y medición de perímetro o volumen del miembro afectado. En el examen clínico destacan el signo de la fóvea para aumento de volumen (edema) y de stemmer para identificar fibrosis subdérmica. Por otro lado, la medición de perímetro se realiza con perimetría o circometría, la cual es una técnica de alta disponibilidad (simple y de bajo costo) (Pereira \& Koshima, 2018; Cuello-Villaverd et al, 2010). La medición de volumen se puede hacer de manera indirecta a través de fórmulas matemáticas basadas en la perimetría o a través de herramientas más sofisticadas como el perómetro. Se considera clínicamente significativo un aumento de $2 \mathrm{~cm}$ o más respecto a la perimetría o diferencia de $200 \mathrm{~mL}$ o más respecto a volumen (Cuello-Villaverd et al, 2010; International Society of Lymphology, 2020).

\section{Riesgo de desarrollo e incidencia del linfedema secundario a cáncer de mama}

La incidencia de linfedema secundario a cáncer de mama dependerá de los distintos factores de riesgo que presenten las personas con este diagnóstico. Los factores de riesgo se pueden dividir en dos grupos, los directos (enfermedad y sus tratamientos) y los indirectos (estilo de vida y características demográficas) (Disipio et al., 2013; Ugur et al., 2013; Fu, 2014; Zhu et al., 2014; Kim et al., 2016) (Tabla 1).
Tabla 1: Factores de riesgo para desarrollar linfedema secundario a cáncer de mama.

\begin{tabular}{l|l}
\hline Factores de riesgo directos & Factores de riesgo indirectos \\
\hline $\begin{array}{l}\text { Disección de linfonodos axilares } \\
\text { Complicaciones del drenaje post-ci- }\end{array}$ & Edad \\
rugía & $\begin{array}{l}\text { Sivel educacional } \\
\text { Radioterapia }\end{array}$ \\
Quimioterapia & $\begin{array}{l}\text { Inactividad física } \\
\text { Hipertensión }\end{array}$ \\
Síndrome de red axilar & $\begin{array}{l}\text { No realizar cuidados preventivos } \\
\text { Mastectomía }\end{array}$ \\
Infección post-cirugía & cirugía \\
\hline
\end{tabular}

Dentro de los factores de riesgo directos, se destaca que a mayor número de linfonodos resecados, mayor es el riesgo de desarrollar linfedema (Senie et al., 2001; Mclaughlin et al., 2008; Fu, 2014; Zhu et al., 2014; Paskett et al, 2015), aunque basta con la remoción de al menos un linfonodo axilar para que el riesgo exista (Kwan et al., 2010). Se ha indicado que la resección de 1 a 15 linfonodos es considerado riesgo bajo, entre 16 a 25 linfonodos se considera riesgo moderado y sobre 26 linfonodos es riesgo elevado (Rebegea et al., 2015). También se ha evidenciado una relación directa entre la cantidad de linfonodos positivos (metastásicos) (Paskett et al., 2015). En contraste, la reconstrucción mamaria inmediata con tejido autólogo puede disminuir el riesgo de generar linfedema en el futuro (Lee et al., 2013). Los factores de riesgo indirecto más relevantes son: la obesidad, el aumento de peso posterior a la cirugía, infecciones o lesiones en el miembro afectado e hipertensión (Fu, 2014; Zhu et al., 2014).

En personas sometidas a extracción de linfonodos axilares, se ha encontrado una incidencia de linfedema variable. Se reporta una incidencia entre $10 \%$ y $49 \%$ para personas con disección de linfonodos axilares, mientras que en personas sometidas a biopsia de linfonodo centinela es entre 5\% y 13\% (Senie et al., 2001; Mclaughlin et al., 2008; Paskett et al., 2015). Parece ser que el riesgo de incidencia de linfedema secundario a cáncer de mama varía en el tiempo después de iniciados los tratamientos médicos. La incidencia de linfedema es elevada en los dos primeros años después de la cirugía (Ozcinar et al., 2012; Disipio et al., 2013; Kim et al., 2019), luego, la incidencia tiene una disminución considerable, que es significativamente menor después de los 5 años después de la cirugía (Disipio et al., 2013).

\section{Prevención y tratamiento del linfedema secundario a cáncer de mama}

Para la prevención de linfedema se han propuesto técnicas quirúrgicas, sin embargo, estas técnicas están indicadas solo en algunos 
casos seleccionados (Pereira \& Koshima, 2018). Otra propuesta de prevención es el tratamiento conservador a través de terapia descongestiva compleja y modelos de atención tempranos y prospectivos. Aunque estos modelos de atención son la estrategia utilizada en países desarrollados, aún falta investigación que fundamente su costo-efectividad (Ramírez et al., 2019).

La terapia descongestiva compleja es el tratamiento gold standard para el linfedema. Esta terapia es una intervención multimodal que consiste en educación acerca de los cuidados de la piel, drenaje linfático manual, ejercicio físico, uso de un sistema elastocompresivo y apoyo psicosocial (Rockson et al., 2019)the pathogenesis of lymphoedema ensues most typically from the modalities employed to stage and treat the cancer (in particular, surgery and radiotherapy. La terapia descongestiva compleja suele ser dividida en fases durante el tratamiento, siendo la fase intensiva la primera y luego la de mantención (Rockson et al., 2019)the pathogenesis of lymphoedema ensues most typically from the modalities employed to stage and treat the cancer (in particular, surgery and radiotherapy. Uno de los pasos fundamentales de la terapia descongestiva compleja es la colocación de un sistema elastocompresivo adecuado (Ciucci et al., 2017). Las prendas de compresión por sí sola se han utilizado con éxito para el tratamiento del linfedema, especialmente en el linfedema relacionado con el cáncer de mama. También han sido efectivos para la prevención en la primera indicación de acumulación de líquido y cambios de volumen mínimo, así como en la etapa inicial del linfedema. Los datos sobre el uso de la prenda sola para las etapas posteriores son muy limitados (International Society of Lymphology, 2020).

\section{Sistema elastocompresivo en linfedema}

Los sistemas elastocompresivos pueden ser divididos en dos grandes grupos, tejido inelástico y tejido elástico. En los sistemas inelásticos encontramos el vendaje multicapa y prendas ajustables con velcro. En los sistemas elásticos encontramos las prendas de tejido circular y plano. Por otro lado, los sistemas elastocompresivos pueden ser clasificados según su clase, es decir, según la presión que ejercen. Las normas más usadas en la clasificación según clase son la alemana (Kwan et al., 2010) y la americana (Goldman et al., 2016) (Tabla 2).

Tabla 2: Clase de compresión en sistemas elastocompresivos, según norma europea y americana.

\begin{tabular}{l|l|l}
\hline Clase de compresión & Estándar Alemán: RAL-GZG 387 & Estándar americano: USA \\
\hline Clase 1 (ligera) & $18-21 \mathrm{mmHg}$ & $15-20 \mathrm{mmHg}$ \\
\hline Clase 2 (moderada) & $23-32 \mathrm{mmHg}$ & $20-30 \mathrm{mmHg}$ \\
\hline Clase 3 (alta) & $34-46 \mathrm{mmHg}$ & $30-40 \mathrm{mmHg}$ \\
\hline Clase 4 (muy alta) & $>50 \mathrm{mmHg}$ & $>40 \mathrm{mmHg}$ \\
\hline
\end{tabular}

Nota: RAL es la abreviación alemana del instituto alemán de garantía de calidad y etiquetado, GZG es la sigla alemana del Centro de Geociencias de la Universidad de Göttingen en Alemania. RAL-GZG 387 es la norma de consenso número 387 entre el RAL y el GZG para compresión. USA es la sigla en inglés de los Estados Unidos de América. La abreviación mmHg es la unidad de presión en milímetros de mercurio.

En el linfedema secundario a cáncer de mama, el vendaje multicapa o las prendas ajustables con velcro se consideran la estrategia más importante de la fase intensiva de la terapia descongestiva compleja y se suelen utilizar durante 4 a 6 semanas para lograr los máximos resultados (Rockson et al., 2019) the pathogenesis of lymphoedema ensues most typically from the modalities employed to stage and treat the cancer (in particular, surgery and radiotherapy. Mientras que las mangas y guantes se utilizan principalmente en la fase de mantención (Rockson et al., 2019)the pathogenesis of lymphoedema ensues most typically from the modalities employed to stage and treat the cancer (in particular, surgery and radiotherapy. En el mercado se ofrecen mangas de tejido plano para tratamiento de linfedema, las que tienen una duración óptima de 24 semanas. Además, se ha propuesto el uso de manga de tejido circular complementada con otras técnicas como ejercicio físico para la prevención (Ochalek et al., 2017).

Se puede observar la evolución de la persona y la efectividad de la terapia con el uso de sistema elastocompresivo a través de la perimetría o perometría. (Cuello-Villaverd et al, 2010). En el caso de la prevención del linfedema, el sistema elastocompresivo será beneficioso al retrasar o evitar la incidencia de linfedema clínicamente detectable, disminuir la sensación subjetiva de pesadez y aumento de volumen. En el caso de linfedema, el efecto del sistema elastocompresivo se evalúa dependiendo de la fase del tratamiento, siendo positivo en la fase de reducción si reduce el perímetro o volumen y la fibrosis del miembro superior afectado, 
mientras que en la fase de mantención debe por lo menos mantener la calidad del tejido y perímetro o volumen (International Society of Lymphology, 2020).

\section{Clasificación de personas en grupos para selección de sistemas elastocompresivos}

Es frecuente que no exista stock suficiente en las centrales de abastecimiento y sea necesario priorizar el uso de los sistemas elastocompresivos. Además, en la actualidad, si bien existe evidencia del uso de las prendas compresivas para prevención y tratamiento del linfedema, no existe un consenso en cuanto a la prenda compresiva a utilizar y queda en manos del equipo de salud especializado su indicación (International Society of Lymphology, 2020). Es por esto que para lograr definir y organizar la entrega de los sitemas elastocompresivos, el equipo de kinesiología de oncología ambulatoria del Complejo Asistencial Dr. Sótero del Río ha propuesto un sistema de selección de la prenda compresiva basándose en un sistema de clasificación en grupos, clasificando a las personas que se trataron por cáncer de mama en dos grandes grupos: personas en prevención de linfedema y personas con diagnóstico de linfedema (Ramírez et al., 2019).

Hasta la actualidad no existen guías o referencias que clasifiquen a las personas tratadas por cáncer de mama en grupos según el riesgo de desarrollar linfedema. Existen nomogramas que utilizan los factores de riesgo para indicar la probabilidad de riesgo para desarrollar linfedema en un determinado límite de tiempo (Bevilacqua et al., 2012; Kim et al., 2019). Un nomograma fácil de utilizar es el propuesto por Bevilacqua JL. y su equipo (Bevilacqua et al., 2012), debido a que cuenta con una versión online
(https://riskcalc.org/BreastCancerArmLymphedema). Sin embargo, a pesar de la existencia de estos nomogramas, la mayoría no considera los nuevos factores de riesgo detectados como el síndrome de red axilar. Por eso, hemos creado un sistema de clasificación clínico que puede ser complementado con los nomogramas.

La clasificación de criterios clínicos tiene 3 etapas. En la primera etapa la paciente será clasificada según la cantidad de linfonodos extirpados. Luego se debe clasificar a la paciente según la presencia o ausencia de factores de riesgo (Tabla 1). Así, aquellas personas que se trataron con biopsia de linfonodo centinela ( $\leq$ 5 linfonodos extirpados) sin factores de riesgo serán clasificadas en el grupo de muy bajo riesgo. Aquellas personas sometidas a biopsia de linfonodo centinela con máximo 2 factores de riesgo y aquellas sometidas a disección de linfonodos axilares hasta grupo II de Berg (6 a 25 ganglios) serán clasificadas en el grupo de bajo riesgo, a menos que presentara más de 2 factores de riesgo, en dicho caso se le clasificaría en el grupo de moderado riesgo. Personas con extirpación de linfonodos hasta nivel III de Berg (sobre 26 ganglios) serán clasificadas en el grupo de alto riesgo, a menos que presenten al menos 2 factores de riesgo adicionales, en dicho caso serán clasificadas en el grupo de muy alto riesgo.

Las personas diagnosticadas con linfedema serán divididas en tres grupos basándonos en la clasificación de la Sociedad Internacional de Linfología (Tabla 3). El grupo 1 de linfedema serán aquellas personas con linfedema estadio I o lla con muy leve fibrosis. El grupo 2 de linfedema serán aquellas personas con linfedema con fibrosis estadio Ilb o III. El grupo 3 de linfedema serán aquellas personas con linfedema maligno por obstrucción tumoral.

Tabla 3: Etapificación del linfedema según la Sociedad Internacional de Linfología.

\begin{tabular}{|c|c|c|}
\hline \multicolumn{2}{|l|}{ Etapa } & Características \\
\hline \multicolumn{2}{|l|}{ Estadío 0} & $\begin{array}{l}\text { Condición latente o subclínica donde la inflamación aún no es evidente a pesar de la alteración en el trans- } \\
\text { porte linfático, cambios sutiles en la composición del líquido tisular. Puede existir meses o años antes de que } \\
\text { se manifieste el edema. }\end{array}$ \\
\hline \multicolumn{2}{|l|}{ Estadío I } & $\begin{array}{l}\text { Representa una acumulación temprana de líquido con un contenido relativamente alto de proteína, que } \\
\text { disminuye con la elevación de la extremidad. Edema con fóvea. }\end{array}$ \\
\hline \multirow{2}{*}{ Estadío II } & Ila (temprano) & La elevación de la extremidad por sí sola rara vez reduce la inflamación del tejido y se produce fóvea. \\
\hline & Illb (tardío) & La extremidad puede o no producir fóvea a medida que se produce un exceso de grasa y fibrosis. \\
\hline \multicolumn{2}{|l|}{ Estadío III } & $\begin{array}{l}\text { Elefantiasis linfostática, donde hay ausencia de fóvea y cambios tróficos de la piel como la acantosis, además, } \\
\text { deposición de grasa y fibrosis, y se pueden desarrollar crecimientos verrugosos. }\end{array}$ \\
\hline
\end{tabular}


Mella et al.

\section{Sistema de clasificación clínico para la selección de sistemas elastocompresivos}

Una vez que se ha clasificado a la persona en el grupo de prevención de linfedema o de linfedema, se debe seleccionar el sistema elastocompresivo según nuestro protocolo (Figuras 1 y 2). En el caso de las personas con muy bajo riesgo, no es necesario indicar un sistema elastocompresivo. Las personas con bajo riesgo se mantendrán bajo vigilancia prospectiva sin sistema elastocompresivo inmediato, sin embargo, en caso de presentar complicaciones, debe ser reasignado. En las personas con moderado, alto y muy alto riesgo se recomienda el uso de mangas de tejido circular. Cabe señalar que cualquier persona en prevención de linfedema que debute con linfedema debe usar un sistema elastocompresivo. La determinación de la compresión a usar (clase I o II) depende de la tolerancia a la compresión y de los síntomas subjetivos que presente la persona, como pesadez, dolor, hinchazón, etc.

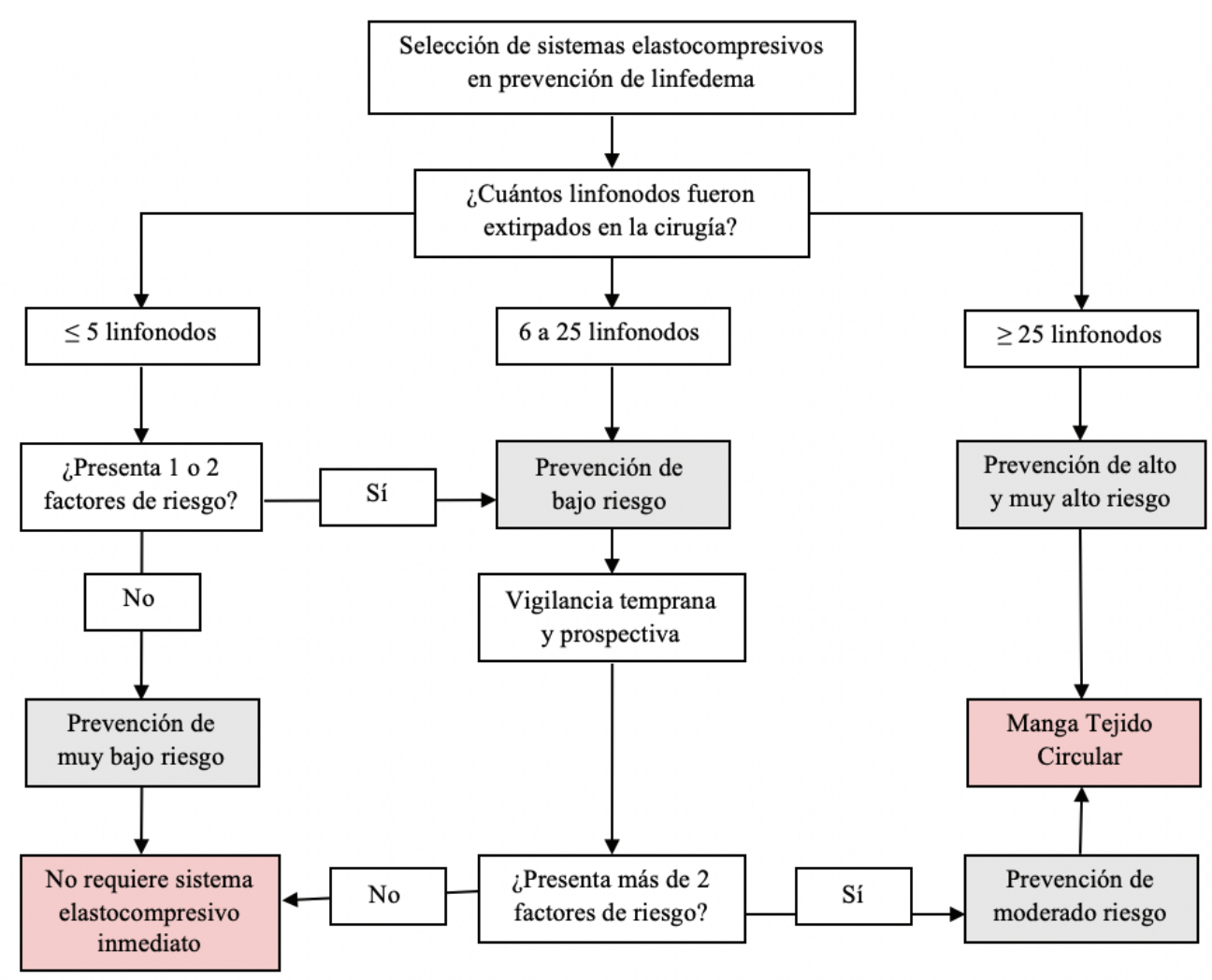

Figura 1: Flujograma para la selección de sistemas elastocompresivos en personas con cáncer de mama para prevención de linfedema.

Las personas con linfedema deben seguir la indicación según la fase de tratamiento dentro de la terapia descongestiva compleja. Las personas del grupo 1 deben hacer uso de manga de tejido plano (clase I o II según tolerancia y/o sintomatología). Las personas del grupo 2 deberán ser tratadas con vendaje multicapa o con prenda ajustable por velcro (con presiones similares a la clase III). Por último, a las personas del grupo 3 no se debe indicar ningún sistema elastocompresivo, a menos que: a) reciba tratamiento paliativo (quimioterapia, hormonoterapia, etc.) y tenga respuesta favorable o b) haya cambios notables en la consistencia del linfedema con drenaje linfático manual. En esos casos seleccionados, se puede indicar vendaje multicapa con precaución, es decir, aplicándolo con presiones similares a la clase I. 
Mella et al.

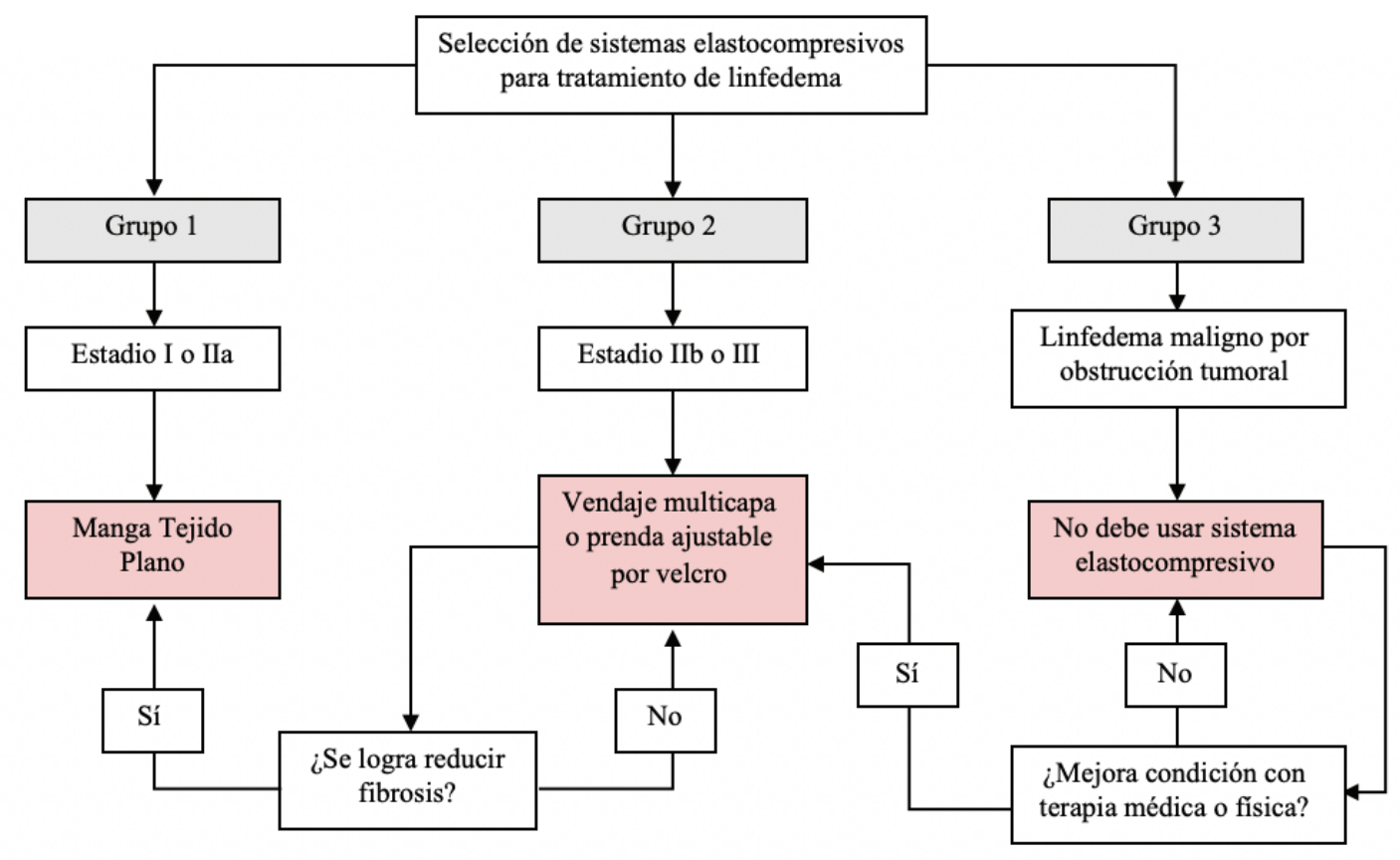

Figura 2: Flujograma para la selección de sistemas elastocompresivos en personas con cáncer de mama para tratamiento de linfedema.

\section{Conclusiones}

El sistema de clasificación mostrado en este artículo puede ser una herramienta fácil y útil para estandarizar la entrega de los sistemas elastocompresivos en servicios públicos como el Complejo Asistencial Sótero del Río. En los servicios públicos los recursos son escasos, por eso, se debe priorizar la entrega de un sistema elastocompresivo en personas con CM. En este artículo no se incluyeron dificultades personales como, por ejemplo, el impedimento en la colocación del sistema elastocompresivo por debilidad muscular, ya que es importante que cada terapeuta utilice su razonamiento clínico. Aún hace falta mayor investigación en cuanto al beneficio aislado de los sistemas elastocompresivos en fases avanzadas del tratamiento para el linfedema, sin embargo, este artículo puede ser un soporte para iniciar protocolos clínicos y eventual investigación.

\section{Financiamiento}

Sin financiamiento.

\section{Conflictos de interés}

Todos los autores de este artículo declaran no tener conflicto de interés

\section{Referencias bibliográficas}

Bevilacqua LB, Kattan MW, Changhong Y. \& Koifman S. (2012). Nomograms for Predicting the Risk of Arm Lymphedema after Axillary Dissection in Breast Cancer. Annals of surgical oncology 19, 2580-2589.

Bray F, Ferlay J. \& Soerjomataram I. (2018). Global Cancer Statistics 2018 : GLOBOCAN Estimates of Incidence and Mortality Worldwide for 36 Cancers in 185 Countries. CA Cancer J Clin. 68, 394- 424.

Ciucci (2017). $6^{\circ}$ Consenso Latinoamericano para el Tratamiento del Linfedema: Guía de tratamiento. Accedido en http://www. centrociucci.com.ar/descargas/6-Consenso-2017-nuevo3.pdf 03 de diciembre de 2019.

Cuello-Villaverde E, Forner-Cordero I. \& Forner-Cordero A. (2010). Linfedema: métodos de medición y criterios diagnósticos. Rehabilitación 44, 21-28.

Disipio T, Rye S, Newman B. \& Hayes S. (2013). Incidence of unilateral arm lymphoedema after breast cancer : a systematic review and meta-analysis. Lancet Oncology 14, 500-515. 
Mella et al.

Fu MR.(2014) Breast cancer-related lymphedema: Symptoms, diagnosis, risk reduction, and management. World J Clin Oncol 5, 241-247.

Goldman MP. \& Weiss RA. (2016). Sclerotherapy E-Book: Treatment of Varicose and Telangiectatic Leg Veins (Expert Consult) (Fifth Edit). Elsevier Health Sciences.

Hidding JT., Beurskens CHG, Van Der Wees PJ, Van Laarhoven HWM. \& Nijhuis-van Der Sanden MWG. (2014). Treatment related impairments in arm and shoulder in patients with breast cancer: A systematic review. PLOS ONE 9.

International Society of Lymphology. The diagnosis and treatment of peripheral lymphedema: 2020 Consensus Document of the International Society of Lymphology.(2020). Lymphology 53, 3-19.

Kim JY, Suh C, Ki Ã, Keum C. \& Sohn ÃJH. (2019). Risk of Lymphedema Following Contemporary Treatment for Breast Cancer: An Analysis of 7617 Consecutive Patients From a Multidisciplinary Perspective. Annals of Surgery $X X(X \mathrm{x})$.

Kim M, Jung S, Kang H, Chung SH, Kim Y, Kim TH. \& Cho KH. (2016). Identification of Prognostic Risk Factors for Transient and Persistent Lymphedema after Multimodal Treatment for Breast Cancer. Cancer Res Treat 48, 1330-1337.

Kwan ML, Darbinian J, Schmitz KH, Citron R, Partee P, Kutner SE. \& Kushi LH. (2010). Risk Factors for Lymphedema in a Prospective Breast Cancer Survivorship Study. Arch Surg 145, 1055-1063.

Lee K, Mun G, Lim S, Pyon J, Oh K. \& Bang S. (2013). The impact of immediate breast reconstruction on post-mastectomy lymphedema in patients undergoing modified radical mastectomy. The Breast 22, 53-57.

Mclaughlin SA, Wright MJ, Morris KT, Giron GL, Sampson MR, Brockway et al. (2008). Prevalence of Lymphedema in Women With Breast Cancer 5 Years After Sentinel Lymph Node Biopsy or Axillary Dissection : Objective Measurements. Journal of Clinical Oncology 26, 5213-5219.

MINSAL (2016). Ministerio de Salud de Chile. Listado de prestaciones específico Régimen de Garantías Explícitas en Salud, Anexo Decreto Supremo N³, de 2016. Gobierno de Chile. Accedido en https:// diprece.minsal.cl/wrdprss_minsal/wp-content/uploads/2018/03/ Lep_incluye-Decreto-8-de-2018.pdf. el 13 agosto 2020.
Ochalek K, Gradalski T. \& Partsch H. (2017). Preventing Early Postoperative Arm Swelling and Lymphedema Manifestation by Compression Sleeves After Axillary Lymph Node Interventions in Breast Cancer Patients : A Randomized Controlled Trial. Journal of Pain and Symptom Management 54, 346-354.

Ozcinar B, Ata S, Kocaman N, Ozkan M, Gulluoglu BM. \& Ozmen V. (2012). Breast cancer related lymphedema in patients with different loco-regional treatments. The Breast 21, 361-365.

Paskett ED, Dean JA, Oliveri JM. \& Harrop JP. (2015). Cancer-Related Lymphedema Risk Factors, Diagnosis, Treatment, and Impact : A Review. Journal of Clinical Oncology 30, 3726-3733

Pereira C.N. \& Koshimal. (2018). Linfedema: actualización en el diagnóstico y tratamiento quirúrgico. Revista Chilena de Cirugía 70, 589-597.

Ramírez-parada, K, Mella-abarca W, Nicoletti-santoni N. \& Aguilera-eguía MPR. (2019). Implementación de un modelo de atención kinesiológico, temprano y prospectivo, en personas con cáncer de mama. Revista Chilena de Cirugía 71, 476-481.

Ramírez K, Acevedo F, Herrera ME, Ibáñez C. \& Sánchez C. (2017). Actividad física y cáncer de mama: un tratamiento dirigido TT Physical activity and breast cancer. Rev. Méd. Chile 145, 75-84.

Rebegea L, Firescu D, Dumitru M. \& Anghel R. (2015). The Incidence and Risk Factors for occurrence of Arm Lymphedema after Treatment of Breast Cancer. Chirurgia (Bucur) 110, 33-37.

Rockson, S. G., Keeley, V., Kilbreath, S., Szuba, A., \& Towers, A. (2019). Cancer-associated secondary lymphoedema. Nature Reviews Disease Primers 5.

Senie RT, Peters M. \& Rosen PP. (2001). Lymphedema in a Cohort of Breast Carcinoma Survivors 20 Years after Diagnosis 1368-1377. Ugur S, Yaprak M, Dolay K. \& Ozmen V. (2013). Risk Factors of Breast Cancer-Related Lymphedema 11, 72-75.

Zhu Y, Xie Y, Liu F, Guo Q, Shen P. \& Tian Y. (2014). Systemic Analysis on Risk Factors for Breast Cancer Related Lymphedema Asian Pac J Cancer Prev. 15, 6535-6541. 